\title{
Lithographically Patterned Channels Spatially Segregate Kinesin Motor Activity and Effectively Guide Microtubule Movements
}

\author{
Samira G. Moorjani, Lili Jia, Thomas N. Jackson, and William O. Hancock* \\ Departments of Bioengineering and Electrical Engineering and Materials Research \\ Institute, The Pennsylvania State University, University Park, Pennsylvania 16802
}

Received January 1, 2003; Revised Manuscript Received March 25, 2003

\begin{abstract}
To extract useful work from biological motor proteins, it is necessary to orient microtubules traveling over kinesin-coated surfaces properly. Toward this goal, we have used microfabrication to construct $1.5-\mu \mathrm{m}$-deep channels in SU-8 photoresist patterned on glass. Although motor proteins bind to all surfaces, these channels localize motility exclusively to the glass surface, and the photoresist creates steep walls that direct microtubule movement. This technique provides a general approach for lithographically patterning enzyme activity.
\end{abstract}

Kinesins are molecular motors that move along microtubules, powering intracellular transport in neurons and other cells. Motor proteins and the microtubule cytoskeleton function as an intracellular railroad system-a railroad with nanometer-scale engines and tracks that transport vesicles and organelles from one location in the cell to another. ${ }^{1,2}$ Because of the importance of organizing and transporting material at nanoscale dimensions, there is considerable interest in harnessing motor proteins for nanotechnology applications. But for this biological system to become technologically useful, methods must be developed to orient microtubules and to direct the motion of microtubules along motor-coated surfaces.

Microtubules are cylindrical polymers of the protein tubulin and are $25 \mathrm{~nm}$ in diameter ${ }^{3}$ and many micrometers long. Kinesins bind to microtubules and use the energy of ATP hydrolysis to walk unidirectionally along these tracks at speeds of nearly $1 \mu \mathrm{m} / \mathrm{s} .{ }^{4}$ The two heads of conventional kinesin alternately step along the microtubule, driving movement, whereas the tail domain binds to intracellular cargo. 5,6 Purified kinesin can be adsorbed to glass and other surfaces and used to transport microtubules across these surfaces, ${ }^{4}$ a geometry termed the microtubule gliding assay. Because individual conventional kinesin molecules can generate forces up to $6 \mathrm{pN}$ (refs 7 and 8 ) and can be adsorbed at densities of at least $10^{3}$ motors $/ \mu \mathrm{m}^{2}$ (ref 9 ), cumulative forces on the order of $\mathrm{nN}$ per $\mu \mathrm{m}^{2}$ are theoretically possible.

The size, efficiency, and potential power density suggest that it is possible to build sophisticated microdevices powered

* Corresponding author. E-mail: wohbio@engr.psu.edu. Phone: 814863-0492. Fax: 814-863-0490. by these motors. These systems may be used in biomolecular separations to isolate molecules of interest from complex mixtures such as cell lysates. The molecules of interest would serve as cargo attached directly or indirectly through other adapter molecules to the microtubules, which would then transport this cargo away from the bulk mixture. More sophisticated devices such as a kinesin-driven microrotor that could be used to drive a pump or a valve in microfluidic applications can also be envisioned.

However, to extract useful work from the kinesin-microtubule system, microtubules must be properly oriented. In previous work, we developed a method for immobilizing oriented microtubules on a surface to create a template for motor-directed assembly or force production. ${ }^{10}$ In the present work, we are inverting the geometry and guiding the movement of microtubules over surface-immobilized motors. The goal of the current work is to establish a general method to target motor activity to specific surfaces and create 3D features that guide microtubule movements along patterned tracks.

There has been some success to date in orienting microtubule motion along modified surfaces. Aligned poly(tetrafluoroethylene) films on glass, ${ }^{11}$ submicrometer steps in silicon wafers, ${ }^{12}$ and micrometer-scale grooves embossed in polyurethane $^{13}$ have been shown to orient microtubule movement from two dimensions to one dimension. However, in all of these cases, the motility is not isolated to the grooves but instead is found everywhere across the surface, hindering the utility of these approaches. Using microfabricated walls patterned with SAL601 photoresist on glass, Hiratsuka et al. were able to localize motility to the glass channels and 
to orient moving microtubules. ${ }^{14}$ Here, we report the use of the epoxy-based photoresist SU-8 to pattern channels on glass. SU-8 is easily available commercially and can be patterned lithographically using near-UV radiation (350$450 \mathrm{~nm}$ ) whereas SAL601 requires electron-beam or deepUV processes, which are more complex and expensive techniques to work with. SU-8 is a high aspect ratio resist capable of producing wall heights ranging from 1 to 200 $\mu \mathrm{m}$ with single spin-coat processes, resulting in steep, perpendicular walls that do not adsorb functional motors and act to redirect microtubule movements.

In preliminary experiments, we tested kinesin motility on glass surfaces spin-coated with a number of different photoresists, including poly(methyl methacrylate) (PMMA), poly(vinyl alcohol) (PVA), AZ5214 (Clariant Corporation, Somerville, NJ), and 1813 (Shipley Company, L. L. C., Marlborough, MA). The goal was to obtain little or no microtubule motility on the resist surface as compared to that on normal glass coverslips. AZ5214 and 1813 were found to be brightly fluorescent and not compatible with the observation of rhodamine-labeled microtubules. With PVA and PMMA, approximately $25 \%$ of the microtubules were seen to be bound to the resist as compared to the value for glass, a minimal difference. In contrast, SU-8 showed very little motility compared to glass; it did not autofluoresce, and it proved ideal for creating high aspect ratio surface features.

To create channels, $1.5-\mu \mathrm{m}$-thick SU-8 2 photoresist was spun onto glass coverslips and patterned using photolithography into channels of widths of 10 and $20 \mu \mathrm{m} .{ }^{15}$ The resulting edges were found to be smooth with little resist residue left behind on the surface after processing, as shown in Figure 1. The patterned coverslips were assembled into flow cells by depositing two strips of grease and $100-\mu \mathrm{m}$ spacers on a standard glass microscope slide and inverting the patterned coverslip to create the top of the chamber. This arrangement permits solution exchange and microscopic observation of the patterned surface. To observe microtubule motility, the surface was first treated with $0.5 \mathrm{mg} / \mathrm{mL}$ casein. Full-length Drosophila conventional kinesin ${ }^{9}$ was then adsorbed to the surface, fluorescent microtubules were washed in along with $1 \mathrm{mM}$ ATP and an antifade cocktail, ${ }^{16}$ and the surface was examined by fluorescence microscopy. To obtain complete differential activity between glass and SU-8, 0.1\% Triton X-100 and $150 \mathrm{mM}$ potassium acetate were included in the motor binding step of the assays. ${ }^{14}$ Triton, a nonionic detergent, was found to eliminate motility on SU-8 but to have no affect on the motility on glass.

Microtubule movements within the channels were visualized by two optical techniques-differential interference contrast (DIC) and fluorescence microscopy using a Nikon E600 microscope (1.3 NA, 100 $\times$ objective) coupled to a Hamamatsu 2400-87 intensified CCD camera and an Argus20 video-processing unit. Images were saved on VHS videotape and analyzed off-line. The simultaneous use of DIC to visualize the channel walls and fluorescence to image the microtubules allowed us to analyze microtubule-wall interactions directly from the video. Figure 2 shows that both the microtubules and the channel walls can be clearly
A

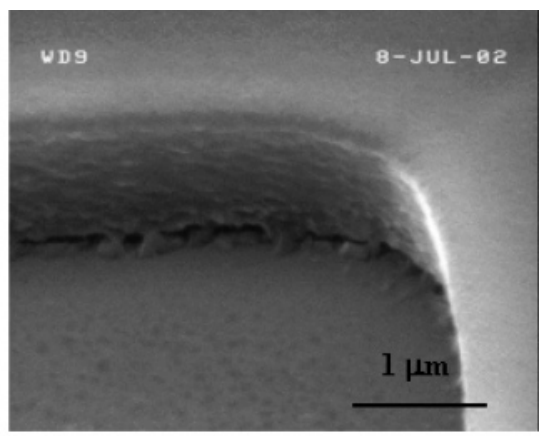

B

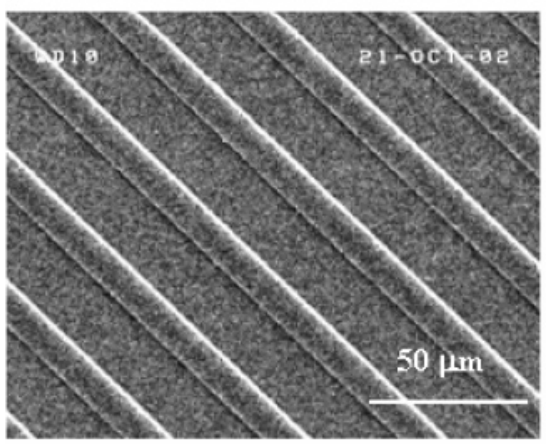

Figure 1. SEM images of microfabricated channels on glass. (A) The edge between the glass and the SU- 8 photoresist shows very little resist residue. The height of the resist wall is $1.5 \mu \mathrm{m}$. (B) Samples used to test motility consisted of a series of channels with widths of $20 \mu \mathrm{m}$ (shown here) and $10 \mu \mathrm{m}$. The walls and channels were visualized by sputtering a $100-\AA$ layer of gold onto the sample and imaging using a Hitachi S-4100 field-emission scanning electron microscope.

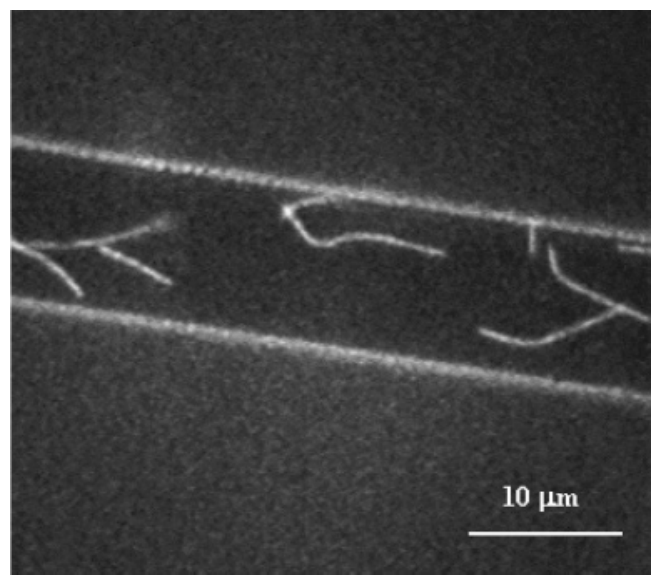

Figure 2. Example of microtubule movement isolated to the glass channel. The microtubules and channels were visualized using simultaneous fluorescence and DIC microscopy. Microtubules bind and move over the glass and not the photoresist.

visualized and demonstrates the complete differential motor activity between the glass in the channel and the SU-8 photoresist.

In addition to localizing the motility exclusively to the exposed glass regions, the steep photoresist sidewalls also acted as effective bumpers to guide microtubules along the channels. Figure 3 shows a sequence of images in which a microtubule collides with the wall, is bent and redirected, and continues moving parallel to the wall. To achieve 


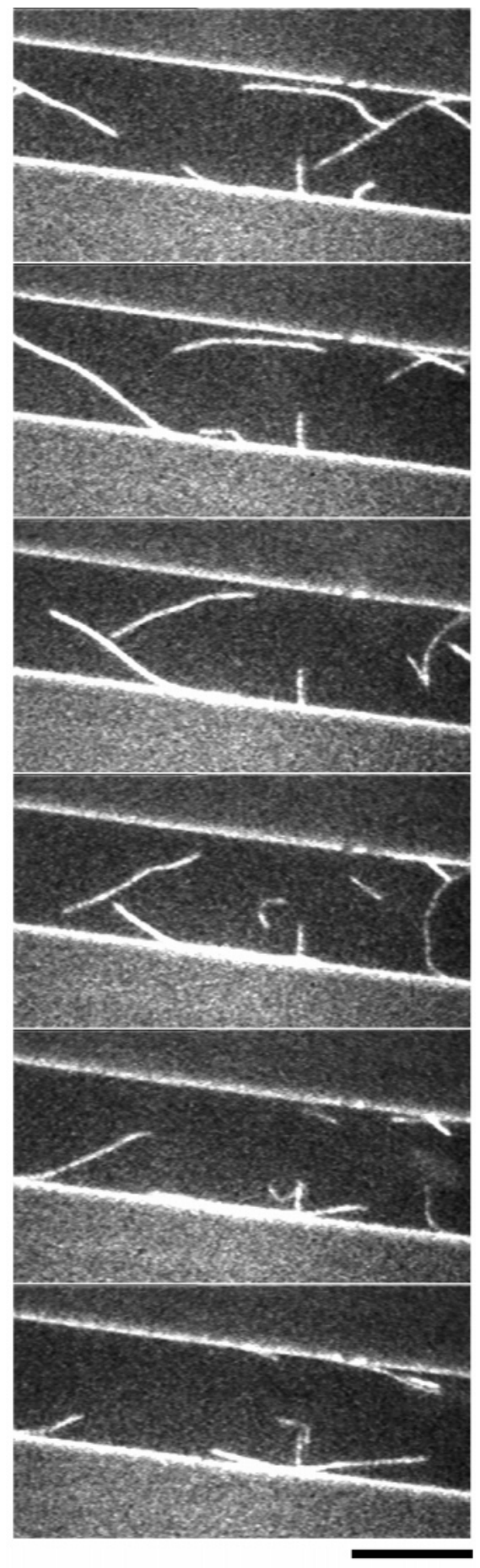

Figure 3. Microtubule being redirected by a microfabricated channel wall. The microtubule moves from left to right in the channel, bumps into the photoresist wall, and is redirected back into the channel. The scale bar at the right is $10 \mu \mathrm{m}$, and images are $12 \mathrm{~s}$ apart. A movie of such motility is included in Supporting Information.

maximal redirection of moving microtubules and minimize the number of microtubules that stalled at wall interfaces, crawled over the wall, or diffused away when they contacted the wall, we chose long microtubules (mean length $9.8 \pm$ $4.5 \mu \mathrm{m} \mathrm{SD}$ ) and a moderate motor concentration (1 $\mathrm{nM}$ active motors ${ }^{16}$ or an estimated 30 motors $/ \mu \mathrm{m}^{2}$ surface density). When much lower motor densities or short microtubules were

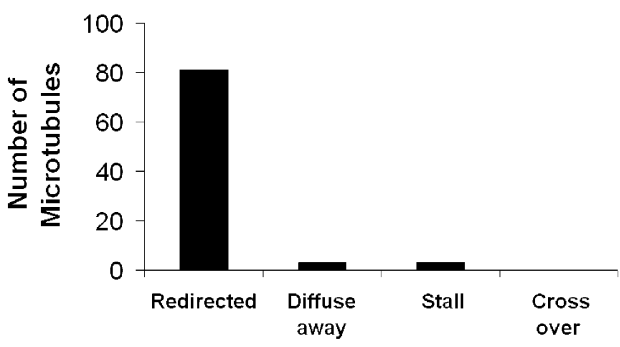

Figure 4. Result of microtubules colliding with microfabricated channel walls. The average microtubule length was $9.8 \mu \mathrm{m}$. Eightyone of the 87 microtubules that were analyzed (93\%) were redirected.

used, microtubules tended to diffuse away when they collided with the wall, and at much higher motor densities, a significant fraction of microtubules simply stalled when they collided with the walls. Long microtubules ensure that the filament is always being moved by a population of motors, and this intermediate motor density ensures that the leading edge of the microtubule is free to bend when it collides with a wall. An analysis of why microtubules do not buckle at higher motor densities is included in Supporting Information.

To analyze in greater detail the effectiveness of the walls in redirecting microtubule movement, we analyzed the result of 87 microtubule-wall collisions and divided them into four categories: (a) the microtubule is properly redirected, (b) the microtubule diffuses away after colliding with the wall, (c) the microtubule stalls when it collides with the wall, and (d) the microtubule climbs over the wall onto the adjacent photoresist. As seen in Figure 4, of the 87 microtubules that were observed, 81 were properly redirected, 3 stalled, 3 diffused away, and none crawled over the wall.

To better understand the interactions, we investigated the effect of the incident angle on the probability of the microtubule being properly redirected. This probability was expected to be high at small incident angles where minimal redirection is required and to fall at sharper angles where more microtubule bending is required. In contrast, as shown in Figure 5, the probability of redirection was virtually independent of the incident angle. Finally, we investigated the path traced by microtubules after collision with the wall; as expected, $86 \%$ of the microtubules were redirected parallel to the wall, and $14 \%$ were reflected back into the channel. Microtubules that collided with the wall at higher incident angles had a slightly lower probability of being redirected back into the channel. (The correlation coefficient between the exit angle and the incident angle was -0.24.)

This excellent performance was the result of both the steepness of the walls and the lack of motor activity on the photoresist. Others have noted that below a certain wall height microtubules climb over walls made from silicon ${ }^{12}$ or polyurethane, ${ }^{13}$ but in both of those cases, there was no differential motor activity on the channel versus the wall and the walls were not vertical.

We next sought to understand why glass supports robust motility whereas the SU-8 photoresist, which was treated with the same casein, kinesin, and microtubule solutions, showed no motility. The lack of motility on SU-8 could be attributed to either motors not binding to the photoresist or 


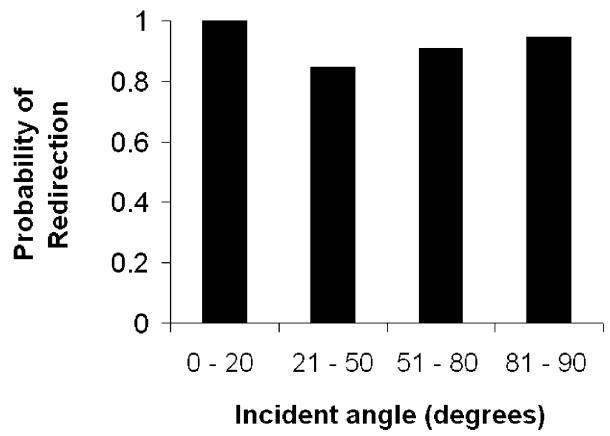

Figure 5. Probability of redirection as a function of the incident angle. For all of the microtubules from Figure 4, the probability of being guided in the same direction of movement is plotted as a function of the incident angle between the microtubule and the channel wall. The probability is largely independent of incident angle. For lower angles, redirected means that the microtubule continues in the same direction in the channel (it does not turn around) whereas for the highest range of angles there was no restriction for the resulting direction following the collision.

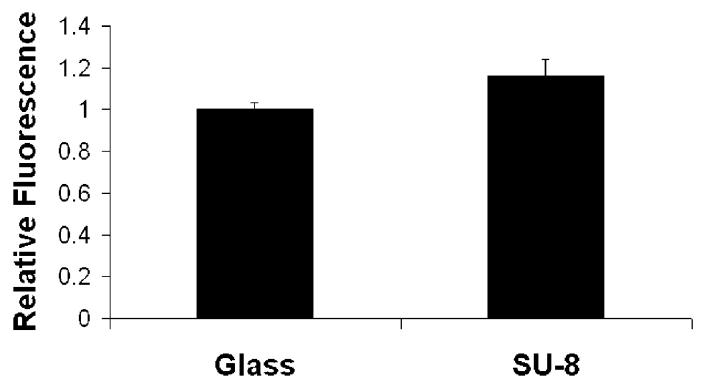

Figure 6. Measure of motor binding to glass and SU-8. Surfaces were incubated in fluorescent kinesin, and the fluorescence intensity on the surface was quantified. A total of 30 determinations were made from three experimental days. The same camera gain, contrast, and brightness levels were used for all samples, and for each coverslip, the background signal in the absence of fluorescent motors was subtracted. Intensity measurements for SU-8 were normalized to the glass values for each day, and the data are presented as mean relative fluorescence \pm SEM. Binding of free fluorophore to both glass and SU-8 surfaces was negligible.

motors binding but being inactivated by the photoresist. To determine the relative amount of motor adsorption to photoresist, we prepared rhodamine-labeled motors using a procedure similar to making rhodamine microtubules. ${ }^{17}$ Using the same motor concentration and solution conditions as for the motility assay, we incubated either glass coverslips or SU-8-treated coverslips with fluorescent motors, washed in antifade cocktail, visualized the surface under fluorescence, saved the image on videotape, and calculated pixel intensities using ScionImage (Scion Corporation, Fredrick, MD). The fluorescence intensity on SU-8 was found to be slightly greater than that of glass (Figure 6), demonstrating that the lack of motility on SU-8 is not due to motors not binding to the surface but instead is due to the fact that the motors must denature on the surface or otherwise be inactivated. This result differs somewhat from the work of Hiratsuka et al., who found that the diminished motility on the SAL601 photoresist was at least partially accounted for by a 2-fold decrease in motor binding to this photoresist. ${ }^{14}$ However, that work used both a different photoresist and a truncated form of kinesin, in contrast to the full-length kinesin used

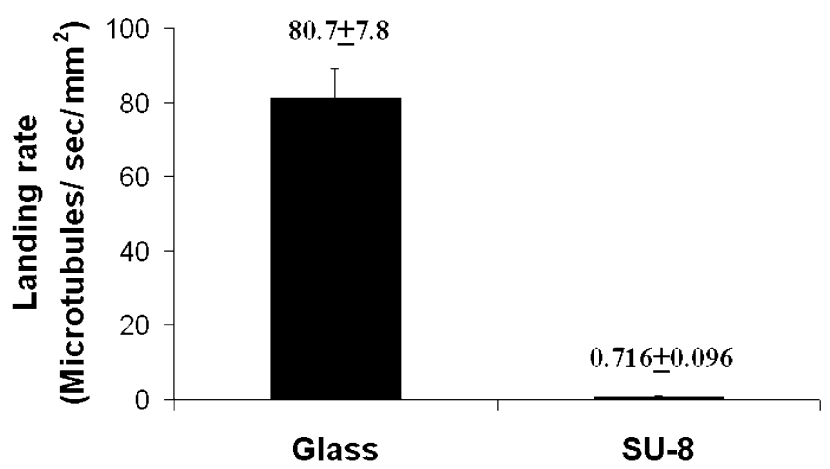

Figure 7. Relative motor activities on glass vs photoresist were compared by measuring the rate that microtubules land on a motorcoated surface in the presence of AMP-PNP. A constant microtubule concentration (32 $\mathrm{nM}$ tubulin) was used, and microtubules were sheared to lengths of $1-5 \mu \mathrm{m}$ by passing the solution twice through a 30-gauge needle. Data were obtained from six screens for glass and eight screens for SU-8 from two experimental days and plotted as mean \pm SEM. The landing rate in the absence of motors was zero for both surfaces; the limits of detection were 0.36 and 0.39 $\mathrm{Mt} / \mathrm{s} / \mathrm{mm}^{2}$ for glass and SU-8, respectively.

here, so the interactions between the motor and the surface are likely different.

After establishing that the lack of motility on the photoresist was due to motor inactivation rather than a lack of motor binding, we set out to quantify the amount of active motor on SU-8 relative to that on glass. Because individual conventional kinesin motors support microtubule gliding, simply observing motility is not a good indicator of the active motor concentration on the surface-motility is observed over at least 3 orders of magnitude of motor surface density. ${ }^{9}$ Instead, the approach to measure motor activity is to determine the rate that microtubules diffuse out of solution and bind to the motor-coated surface. This is analogous to measuring the rate of a second-order reaction, where the rate is proportional to the concentration of active motors on the surface. It has been shown that below a maximal motor density, where the landing rate is limited by the rate that microtubules diffuse to the surface, the landing rate is indeed proportional to motor density..$^{9,18,19}$

Using identical microtubule and motor concentrations, we compared microtubule landing rates on glass and SU-8 photoresist in the presence of the nonhydrolyzable ATP analogue AMP-PNP, which promotes strong binding of kinesin to microtubules. ${ }^{20}$ The landing rate was reduced by greater than 100-fold on SU-8 compared to that on glass (Figure 7). Hence, although motor activity is not entirely eliminated on the photoresist, the density of active motors on SU-8 is so low that there are only transient interactions of microtubules with the surface. The glass, in contrast, contains a high density of active motors on the surface, which ensures that as a microtubule travels over the surface it always encounters new motors and hence rarely detaches from the surface.

It is not entirely clear why glass supports robust motility but SU-8 does not. Although glass is a hydrophilic surface, untreated SU-8 is more hydrophobic (the contact angle with water was found to be $64^{\circ}$ ), and it is conceivable that this hydrophobicity leads to motor denaturation on the surface. Consistent with this, during the course of these experiments, 
we tried etching SU-8 photoresist with $\mathrm{O}_{2}$ or $\mathrm{CF}_{4}$ plasma and found that these treatments both decreased the hydrophobicity of the surface (contact angle fell to $0^{\circ}$ ) and resulted in robust motility. It should be noted that for all motility experiments surfaces are treated with casein before and during motor binding; it has been shown that in the absence of such pretreatment motors inactivate on glass surfaces until a concentration roughly equal to a surface monolayer is reached, and only then are surface-bound motors functional. ${ }^{4}$ It is presumed that casein passivates glass surfaces sufficiently to prevent motor denaturation on glass but does not block the surface sufficiently to prevent motor binding. Though it cannot be ruled out that the kinesin tails actually bind to the surface-adsorbed casein instead of to the glass itself, the fact that casein present in solution does not affect motor binding argues against this. The reason that casein passivates glass sufficiently to allow motility but does not passivate SU-8 is the subject of ongoing work that is important both in understanding the biophysics of kinesin motility on surfaces and for applications that seek to harness kinesin motility for biotechnological uses.

The demonstrated differential motor activity provides a novel approach to patterning proteins on surfaces. Current methods of patterning proteins such as soft lithography used to pattern different surface chemistries, ${ }^{21,22}$ liftoff techniques, ${ }^{23}$ dip-pen nanolithography, ${ }^{24}$ or directly stamping proteins onto functionalized surfaces ${ }^{25}$ rely on adsorbing proteins to specific regions of a surface and not to other regions. The lithographic approach employed here did not alter the degree of motor adsorption but nonetheless created dramatic spatial differences in functional activity. A similar approach to creating high spatial resolution patterns of protein activity as opposed to patterns of protein concentration could conceivably be used to pattern enzymes for biosensors or cell adhesion proteins for applications utilizing spatial arrays of cells.

In conclusion, we present a novel technique for patterning functional kinesin motors on surfaces and creating channels to guide the direction of moving microtubules, a necessary step toward extracting useful work from the microtubulemotor system. We are currently working toward creating unidirectional movement of microtubules by designing different channel geometries, with the goal of harnessing this movement for biotransport systems in which molecules of interest are attached to microtubules and carried unidirectionally along fabricated channels to the site of interest. These systems may then be integrated into microchips to create devices for microscale separation of minute biological samples.

Acknowledgment. This work was supported by the Whitaker Foundation (to W.O.H.) and the ONR and DARPA (to T.N.J.). We thank Adam Stine, Victoria Lee, and Yangrong Zhang for help in purifying and quantitating kinesin motors and Chung-Chen Kuo for lithography assistance.

Supporting Information Available: An analysis of the dependence of microtubule buckling on motor density and a movie illustrating the alignment of microtubules on collision with the wall. This material is available free of charge via the Internet at http://pubs.acs.org.

\section{References}

(1) Howard, J. Annu. Rev. Physiol. 1996, 58, 703-729.

(2) Hirokawa, N. Science (Washington, D.C.) 1998, 279, 519-526.

(3) Nogales, E.; Whittaker, M.; Milligan, R. A.; Downing, K. H. Cell 1999, 96, 79-88.

(4) Howard, J.; Hudspeth, A. J.; Vale, R. D. Nature (London) 1989, 342, 154-158.

(5) Hirokawa, N.; Pfister, K. K.; Yorifuji, H.; Wagner, M. C.; Brady, S. T.; Bloom, G. S. Cell 1989, 56, 867-878.

(6) Yang, J. T.; Saxton, W. M.; Stewart, R. J.; Raff, E. C.; Goldstein, L. S. Science (Washington, D.C.) 1990, 249, 42-47.

(7) Svoboda, K.; Schmidt, C. F.; Schnapp, B. J.; Block, S. M. Nature (London) 1993, 365, 721-727.

(8) Meyhofer, E.; Howard, J. Proc. Natl. Acad. Sci. U.S.A 1995, 92, $574-578$.

(9) Hancock, W. O.; Howard, J. J. Cell Biol. 1998, 140, 1395-1405.

(10) Brown, T. B.; Hancock, W. O. Nano Lett. 2002, 2, 1131-1135.

(11) Dennis, J. R.; Howard, J.; Vogel, V. Nanotechnology 1999, 10, 232236.

(12) Stracke, R.; Bohm, K. J.; Burgold, J.; Schacht, H.-J.; Unger, E. Nanotechnology 2000, 11, 52-56.

(13) Hess, H.; Clemmens, J.; Qin, D.; Howard, J.; Vogel, V. Nano Lett. 2001, 1, 235-239.

(14) Hiratsuka, Y.; Tada, T.; Oiwa, K.; Kanayama, T.; Uyeda, T. Q. Biophys. J. 2001, 81, 1555-1561.

(15) Coverslips (Corning No. $1 \frac{1}{2}, 18 \mathrm{~mm}$ ) were prebaked at $200{ }^{\circ} \mathrm{C}$ for 5 min on a hot plate, and SU-8 2 films (MicroChem Corp., Newton, MA) were spin cast at $3000 \mathrm{rpm}$ for $30 \mathrm{~s}$, followed by a two-step soft bake at $65^{\circ} \mathrm{C}$ for $1 \mathrm{~min}$ then at $95^{\circ} \mathrm{C}$ for $1 \mathrm{~min}$. The coverslips were then irradiated with 436-nm light through a photo mask, baked at $65^{\circ} \mathrm{C}$ for $1 \mathrm{~min}$ and $95^{\circ} \mathrm{C}$ for $1 \mathrm{~min}$, developed in SU-8 developer, rinsed briefly with isopropyl alcohol, and dried with a gentle stream of $\mathrm{N}_{2}$.

(16) The full-length, bacterially expressed Drosophila kinesin heavy chain was purified as previously described. ${ }^{9}$ Tubulin was purified from bovine brain ${ }^{26}$ and fluorescently labeled with rhodamine. ${ }^{17}$ BRB80 buffer ( $80 \mathrm{mM}$ PIPES, $1 \mathrm{mM}$ EDTA, $1 \mathrm{mM} \mathrm{MgCl}_{2}$, $\mathrm{pH}$ 6.9) was used for all solutions. Motors $(3.7 \mathrm{mg} / \mathrm{ml}$ total protein concentration determined from Coomassie-stained gels or $1 \mathrm{nM}$ active motor concentration determined by radionucleotide binding ${ }^{27}$ ) were adsorbed to surfaces in the presence of $0.2 \mathrm{mg} / \mathrm{mL}$ casein, $0.1 \mathrm{mM} \mathrm{ATP}, 0.1 \%$ Triton X-100, and $150 \mathrm{mM}$ potassium acetate. After a 5-min incubation, taxol-stabilized microtubules $(32 \mathrm{nM}$ tubulin concentration, length $5-20 \mu \mathrm{m}$ ) were infused in the presence of $1 \mathrm{mM} \mathrm{ATP}$, $0.2 \mathrm{mg} / \mathrm{mL}$ casein, $10 \mu \mathrm{M}$ taxol, and an antifade cocktail $(20 \mathrm{mM}$ D-glucose, $0.02 \mathrm{mg} / \mathrm{mL}$ glucose oxidase, $0.008 \mathrm{mg} / \mathrm{mL}$ catalase, $0.5 \%$ $\beta$-mercaptoethanol).

(17) Hyman, A. A. J. Cell Sci. Suppl. 1991, 14, 125-127.

(18) Stewart, R. J.; Semerjian, J.; Schmidt, C. F. Eur. Biophys. J. 1998, $27,353-360$.

(19) Marcus, A. I.; Ambrose, J. C.; Blickley, L.; Hancock, W. O.; Cyr, R. J. Cell Motil. Cytoskeleton 2002, 52, 144-150.

(20) Lasek, R. J.; Brady, S. T. Nature (London) 1985, 316, 645-647.

(21) Prime, K. L.; Whitesides, G. M. Science (Washington, D.C.) 1991, $252,1164-1167$.

(22) Mrksich, M.; Whitesides, G. M. Annu. Rev. Biophys. Biomol. Struct. 1996, 25, 55-78.

(23) Ilic, B.; Craighead, H. G. Biomed. Microdevices 2000, 2, 317-322.

(24) Lee, K. B.; Park, S. J.; Mirkin, C. A.; Smith, J. C.; Mrksich, M. Science (Washington, D.C.) 2002, 295, 1702-1705.

(25) Kane, R. S.; Takayama, S.; Ostuni, E.; Ingber, D. E.; Whitesides, G. M. Biomaterials 1999, 20, 2363-2376.

(26) Williams, R. C., Jr.; Lee, J. C. Methods Enzymol. 1982, 85 Pt. B, $376-385$.

(27) Coy, D. L.; Wagenbach, M.; Howard, J. J. Biol. Chem. 1999, 274, 3667-3671.

NL034001B 